\title{
Sub-nanosecond switching and acceleration to relativistic energies of field emission electron bunches from metallic nano-tips
}

\author{
S. Tsujino, ${ }^{1, a)}$ M. Paraliev, ${ }^{2}$ E. Kirk, ${ }^{1}$ C. Gough, ${ }^{2}$ S. Ivkovic, ${ }^{2}$ and H.-H. Braun ${ }^{3}$ \\ ${ }^{1}$ Laboratory for Micro- and Nanotechnology, Department of Synchrotron Radiation and Nanotechnology, \\ Paul Scherrer Institut, CH-5232 Villigen-PSI, Switzerland \\ ${ }^{2}$ Department of Large Scale Research Facilities, Paul Scherrer Institut, CH-5232 Villigen-PSI, Switzerland \\ ${ }^{3}$ Swiss FEL, Paul Scherrer Institut, CH-5232 Villigen-PSI, Switzerland
}

(Received 18 April 2011; accepted 4 May 2011; published online 10 June 2011)

\begin{abstract}
The authors explore the generation of short electron bunches from a field-emitter array cathode consisting of nanometer-sized metallic tips that is compatible with an acceleration electric field above $10 \mathrm{MV} / \mathrm{m}$. Sub-nanosecond field emission electron bunches were generated by applying fast electrical pulses to an on-chip electron extraction gate electrode of the cathode. The subsequent acceleration of the field emission electron bunches to $5 \mathrm{MeV}$ was demonstrated using the combined diode-RF cavity SwissFEL electron gun test facility. (C) 2011 American Institute of Physics. [doi:10.1063/1.3594579]
\end{abstract}

The high current density and high brightness of field emission beams ${ }^{1-3}$ have motivated the use of field emitters as electron sources in applications such as microwave amplifiers $^{4-8}$ and free electron lasers. ${ }^{2,3,9-18}$ For the anticipated current densities, a high acceleration gradient is important to preserve the beam emittance. Nano-fabricated field emitter array (FEA) cathodes, equipped with nanometer-scale sharp tips and an on-chip electron extraction gate electrode, are well-suited for this purpose, owing to partial electrostatic shielding of individual field emitters from the accelerating field by the gate electrode, and the capability of switching the electron emission independently from the accelerating field. Recently, the switching of a field-emission electron beam from a FEA on a time scale of a few nanoseconds and up to $300 \mathrm{keV}$ acceleration of the field-emission electron beam by a diode-gun accelerator was reported. ${ }^{18}$ In this work, we show field emission current switching for subnanosecond electron bunch generation and the combination of the method with a diode-radio frequency (RF) cavity electron gun to generate field-emission electron beam with relativistic energies.

The single-gate FEA cathodes (Fig. 1) were fabricated by molding and a self-aligned gate process. ${ }^{15}$ They consist of $1.2 \times 10^{5}$ pyramidal shape molybdenum field emitter tips $\sim 1.2 \mu \mathrm{m}$ in height and a $1.5 \mu \mathrm{m}$ base size, aligned with $5 \mu \mathrm{m}$ spacing on a $0.3 \mathrm{~mm}$ thick electroplated nickel substrate. The electron emission is switched on by applying a negative voltage pulse, $-V_{\mathrm{em}}$, to the field emitters with respect to the electron extraction gate electrode, which has $\sim 2 \mu$ m diameter apertures for individual emitters. The gate electrode is a 0.5 $\mu$ m-thick molybdenum film separated from the emitter substrate by a $1.2-\mu$ m-thick $\mathrm{SiO}_{2}$ film, and is patterned to a 5.4mm-diameter circular shape. The gate-electrode diameter here is required to apply a large accelerating electric field above 10 $\mathrm{MV} / \mathrm{m} .{ }^{18}$ Consequently, it leads to an increased capacitance,

\footnotetext{
${ }^{a}$ Author to whom correspondence should be addressed. Electronic mail: soichiro.tsujino@psi.ch.
}

$C_{\mathrm{g}}$, between the gate electrode and the field emitter substrate. To partly compensate for the capacitance increase, we inserted a 1.2- $\mu$ m-thick $\mathrm{SiON}$ film underneath the $\mathrm{SiO}_{2}$ layer outside the emitter array area, as shown in Fig. 1(b). The resulting $C_{\mathrm{g}}$ was $\sim 0.5 \mathrm{nF}$. This is an order of magnitude larger than that of a Spindt FEA used for the RF emission-gate experiment ${ }^{7}$ due to the larger gate electrode area. However, the $R C$ constant of our all-metallic FEAs is several orders of magnitude below $1 \mathrm{~ns}$, since the resistance of the substrate is low. ${ }^{18}$ Hence, it is possible to switch $V_{\text {em }}$ in $\sim 1$ ns [in full width at half maximum (FWHM)] by using the following method [cf., Figs. 1(c) and 1(d)]: first we apply a negative current pulse to the emitter substrate with the FWHM-duration, $T_{\mathrm{p}}$, below $1 \mathrm{~ns}$, with the amplitude determined by $C_{\mathrm{g}} V_{\mathrm{em}} / T_{\mathrm{p}}$; then we apply a second current pulse with positive polarity, and with the same duration and amplitude, delayed by $\Delta T$ of $1 \mathrm{~ns}$ from the first pulse. As a result, combined with the nonlinear current-voltage characteristics of the field emission, ${ }^{7}$ we expect to be able to produce a field emission current pulse with sub-nanosecond duration. In the experiment described in the following text, we used current pulses with $T_{\mathrm{p}}$ of 0.65 ns. We also adjusted $V_{\text {em }}$ by applying a dc bias, $V_{\mathrm{dc}}$, in series to the double current pulses.

The electron bunch acceleration experiment used the combined diode-RF cavity gun [Fig. 1(c)] of the SwissFEL test facility at the Paul Scherrer Institut. In the diode gun, a pulsed cathode voltage up to $-500 \mathrm{kV}$ with $250 \mathrm{~ns}$ duration (FWHM) and a maximum repetition frequency of $10 \mathrm{~Hz}$ can be applied by using the critically coupled air-core transformer technology. ${ }^{19}$ After passing through the anode iris, the electron bunch is transported to a 2-cell RF-cavity through a $150 \mathrm{~mm}$ tunnel and further accelerated by a $1.49896 \mathrm{GHz}$ RF-field. ${ }^{20}$ The electron beam is detected either by one of the scintillating screen monitors or by a Faraday cup. The energy of the accelerated electron beam can be measured by dispersing the beam using a dipole magnet located $3 \mathrm{~m}$ downstream of the beam line, and imaging it on a screen monitor. 

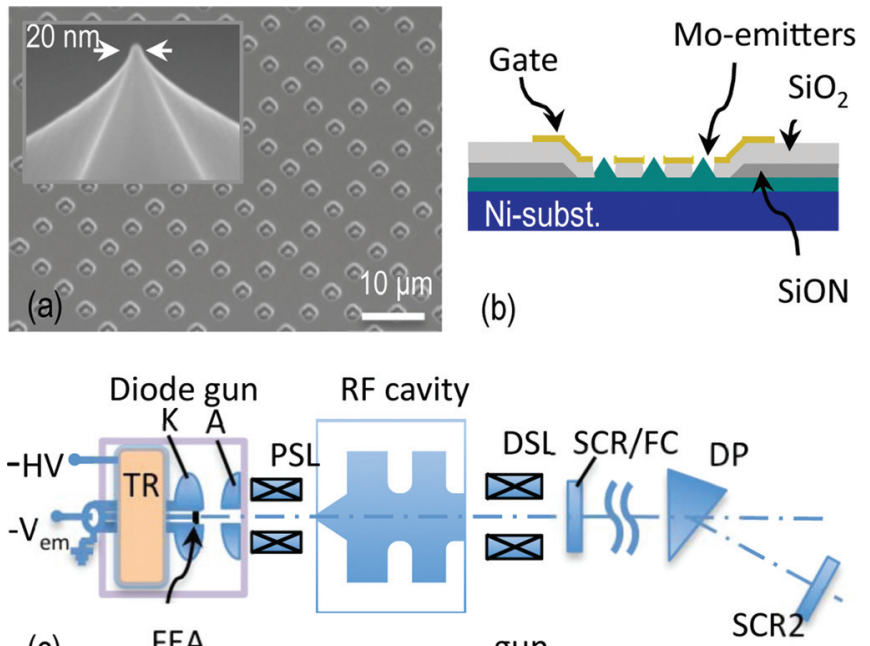

(c)

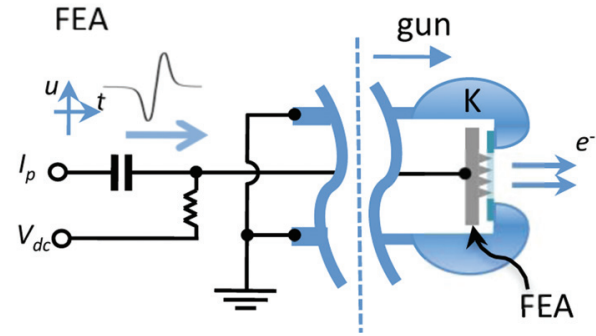

FIG. 1. (Color online) (a) Scanning electron micrograph of a 5- $\mu$ m-pitch molybdenum FEA. The inset shows the high-resolution picture of one emitter. (b) Schematic cross-section of an FEA chip. (c) Schematic diagram of combined diode-RF cavity electron gun. Only relevant parts are shown. K: cathode holder; A: anode,; TR: transformer; PSL: pulsed solenoid; DSL: solenoid; SCR and SCR2: scintillating screen monitor; FC: Faraday cup; DP: dipole magnet. SCR and FC can be moved in/out to/from the beam axis. (d) Schematic diagram of the FEA driver and the FEA holder (HV circuit is not included).

To integrate a FEA in the diode-RF cavity gun, we developed a holder with a low-inductance electrical contact [K in Fig. 1(d)]. ${ }^{18}$ In this scheme, we apply the FEA driving pulse via a 22-m-long semi-rigid coaxial cable, which also forms the secondary coil of the high voltage transformer. A spring-loaded contact makes the electrical connection to the FEA substrate. The cathode holder has a $4 \mathrm{~mm}$-diameter opening at the center in order to expose the FEA to the diode acceleration field. The gate electrode is contacted to the holder at the $0.5-\mathrm{mm}$-wide edge of the opening. Due to the length of the coaxial cable, up to $0.2 \pm 0.1 \mathrm{~ns}$ broadening of the driving pulse is expected.

In the experiment, we first installed the FEA chip in a preparation chamber for processing by dc bias ${ }^{18,21}$ and pulsed current tests. The processing lasted for a few days until the gradual increase of current at a given bias saturates and stable current-voltage characteristics are obtained. In this chamber, the anode was a stainless steel Faraday cup at a $10 \mathrm{~mm}$ distance from the FEA, the maximum anode voltage was limited to $4 \mathrm{kV}$, and the base pressure was $5 \times 10^{-10}$ mbar. After the processing, the FEA chip was transferred to the diode-gun through air within $\sim 5 \mathrm{~min}$. Because of the transfer through the air, a second processing in the diode-gun was necessary. This began when the gun vacuum reached the base pressure of $\sim 1 \times 10^{-8}$ mbar and continued for a few days. We found that less time was required than the first
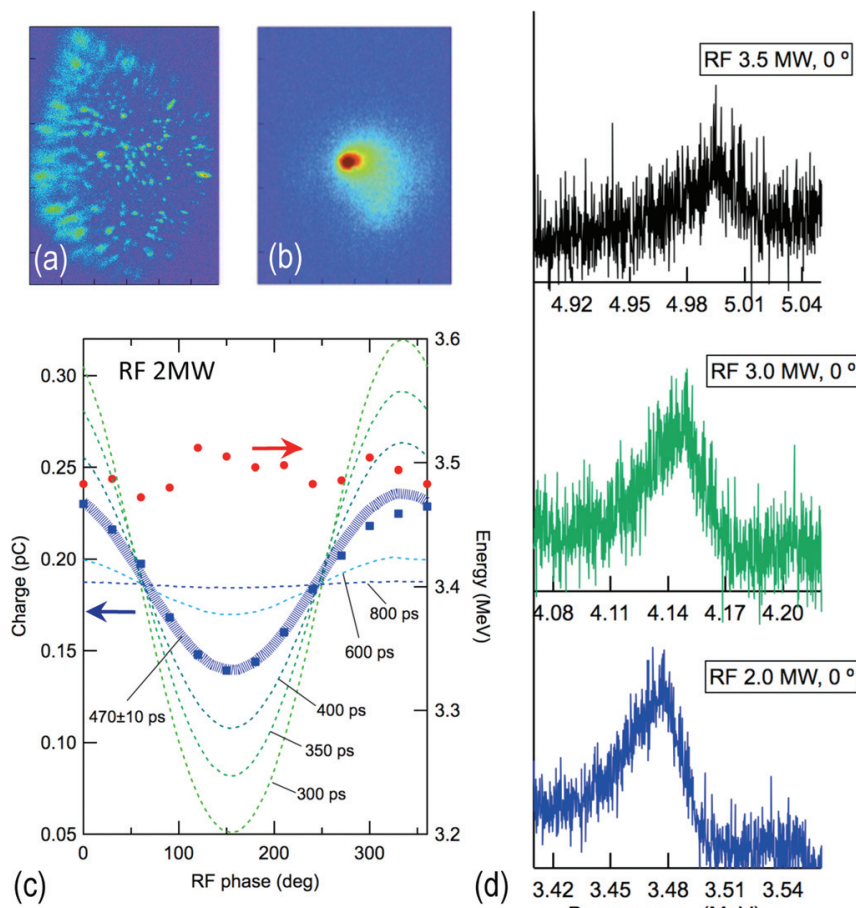

(d) $\begin{array}{lllll}3.42 & 3.45 & 3.48 & 3.51 & 3.54\end{array}$

Beam energy (MeV)

FIG. 2. (Color online) Beam image of field emission electron pulse (a) accelerated to $200 \mathrm{keV}$ by diode gun, and (b) accelerated by combined diode-RF cavity gun to $3.5 \mathrm{MeV}$. (c) RF phase dependence of the bunch charge (filled squares) and beam energy (filled circles). Curves are the simulated RF phase dependence of the bunch charge calculated with different bunch durations between 300 and 800 ps. (d) Energy spectra of the beam accelerated by three different RF power levels.

processing process in order to recover $\sim 30 \%$ of the current $\left(1.8 \mathrm{~mA}\right.$ at $V_{\mathrm{em}}$ of $\left.63 \mathrm{~V}\right)$ obtained in the preparation chamber.

In Fig. 2(a), we show the field emission beam accelerated only by the diode gun to $200 \mathrm{keV}$ and imaged on the scintillating screen monitor (SCR). Under these conditions, the FEA emitted $0.46 \mathrm{pC}$ bunch charge when a double pulse current with an amplitude of $\sim 77 \mathrm{~A}$ was applied with a reverse dc bias of $+12.5 \mathrm{~V}$. The acceleration gradient on the FEA surface was $14 \mathrm{MV} / \mathrm{m}$. The granular emission pattern suggests an inhomogeneous current distribution among the emitters as previously observed. ${ }^{18}$ In the next step of the experiment, we supplied RF power to the RF cavity and further accelerated the electron bunch by the RF cavity. Figure 2(b) shows the beam image focused on the SCR when the RF power was $2 \mathrm{MW}$ and the relative phase of the RF acceleration field was equal to $300^{\circ}$ [see Fig. 2(c) and the following text]. In this case, it was difficult to resolve the detail of the field-emission beam as in Fig. 2(a).

In Fig. 2(c), we show the RF phase dependence of the electron bunch charge (left axis) and the beam energy (right axis). The sinusoidal variation of the bunch charge when the $\mathrm{RF}$ phase was varied from 0 to $360^{\circ}$ shows that the FWHM duration, $T_{\mathrm{FEA}}$, of the field emission current pulse is shorter than one RF cycle of $0.667 \mathrm{~ns}$, since only the electrons accelerated in the propagation direction can pass through the cavity. The $<100 \%$ modulation of the bunch charge with the RF phase shows that $T_{\text {FEA }}$ is longer than one-half the RF cycle of $0.334 \mathrm{~ns}$. To quantitatively evaluate $T_{\mathrm{FEA}}$, we simulated 
the electron bunch transmission through the RF-cavity assuming a Gaussian current pulse. We considered that electrons transmit the RF-cavity when the RF electric field is along the propagation direction. The variation of the overlap in time between the forward RF field and the current pulse with the duration shorter than one RF cycle resulted in the RF phase dependence of the bunch charge. The curves in Fig. 2(c) show the calculated RF phase dependence for $T_{\mathrm{FEA}}$ to be between 0.3 and 0.8 ns. Good agreement between theory and experiment was obtained for $T_{\mathrm{FEA}}$ of $0.47 \pm 0.01$ ns. We also note that the average transmitted bunch charge of $0.19 \mathrm{pC}$ was slightly below one-half of the bunch charge $(0.46 \mathrm{pC})$ without the RF acceleration because of the energy dispersion of the transmitted accelerated electrons and the resultant defocusing effect. In the simulation, we included this effect, which is approximately the same as setting unity transmission when the forward RF electric field is above $30 \%$ of the maximum value, although the defocusing effect influences the estimated value of $T_{\mathrm{FEA}}$ by less than $4 \%$.

To measure the energy spectrum of the accelerated beam, we next transported it down to the dipole magnet [DP in Fig. 1(c)] and imaged the dispersed beam on the SCR2. The results at three RF powers with a fixed phase of $0^{\circ}$ are shown in Fig. 2(d). We found that the beam energy was in the relativistic range as expected: when the RF power was increased from 2.0 to $3.5 \mathrm{MW}$, the peak beam energy increased from $3.48 \mathrm{MeV}$ up to $5.0 \mathrm{MeV}$. The increasing broadening of the spectra with the beam energy, as suggested in the observed spectra, is likely due to the increased defocusing because of the energy dispersion of the beam.

As a reference, we also measured the field emission pulse in the preparation chamber by applying an anode bias voltage, $V_{\mathrm{a}}$, between $20 \mathrm{~V}$ and $4 \mathrm{kV}$. The current pulses were split via a bias-T of $2 \mathrm{GHz}$-bandwidth and were detected by a digital oscilloscope with a $3 \mathrm{GHz}$ bandwidth and $10 \mathrm{GS} / \mathrm{s}$. Figure 3 shows the results. The pulse shape exhibited an effect of the electron transit time from the FEA to the anode, which was evidenced by the decreasing current peak maxi-
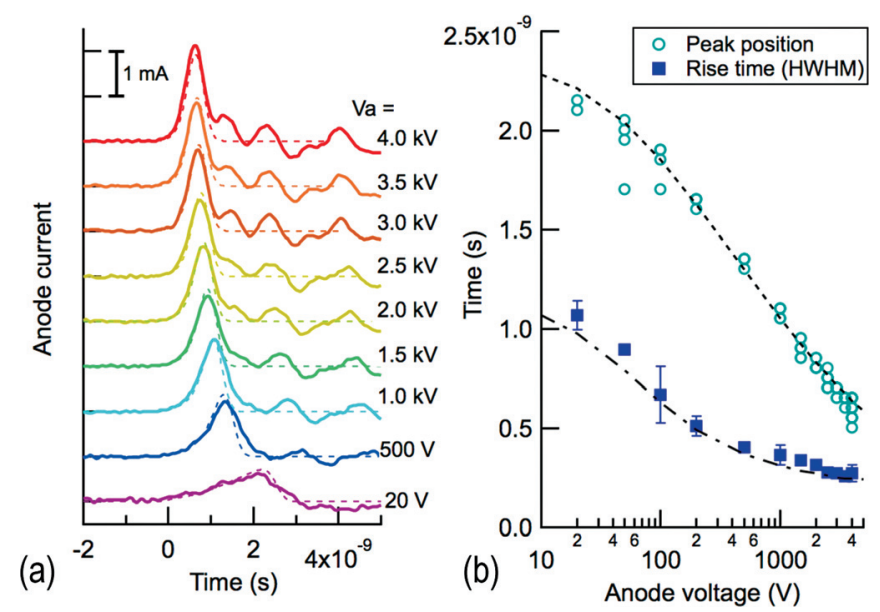

FIG. 3. (Color online) (a) Field emission current pulses at different anode voltages, $V_{\mathrm{a}}$, measured in a preparation chamber. Broken curves show the theory. (b) Peak position time and the rise time in half-width at the half-

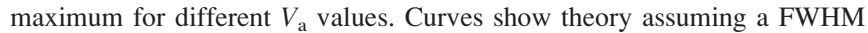
duration of the field emission current pulse, $T^{(\mathrm{th})}$, of $0.4 \mathrm{~ns}$. mum time, $\tau$, with the increase of $V_{\mathrm{a}}$ and by the narrowing of the pulse duration at the same time. In Fig. 3(b), we display $\tau$ and the rise time, $\delta \tau$ (we defined $\delta \tau$ as the difference of $\tau$ and the half-width at half-maximum time) as a function of $V_{\mathrm{a}}$. The smallest $\delta \tau$ of $0.26 \mathrm{~ns}$ was observed for $V_{\mathrm{a}}$ of $4 \mathrm{kV}$. The $V_{\mathrm{a}}$-dependence of $\delta \tau$ was correlated to that of $\tau$ due to the induced current of the propagating field emission beam. ${ }^{18,22,23}$ By assuming a Gaussian shape field emission pulse and convoluting it with the effect of the induced current in the measurement geometry, including the effect of an angular divergence of $\sim 60^{\circ}$ at the emitter tip, ${ }^{14,16,17}$ we calculated the current pulse waveform. The result is compared with the experiment by the curves in Figs. 3(a) and 3(b). A good agreement between theory and experiment was obtained when we assumed the FWHM duration of the field emission current pulse, $T^{\text {(th) }}$, of $0.4 \pm 0.1 \mathrm{~ns}$. This value, which was compatible within $\sim 0.1 \mathrm{~ns}$ with $T_{\mathrm{FEA}}$ evaluated from the RF phase scan and with $\delta \tau$, confirmed that the pulse duration of the field emission current from our FEA was indeed in the sub-nanosecond range. One lower limit to the field emitter switching time comes from the diffusion of the switching current pulses from the edge to the center of the array. For the FEA used in the present work, it is equal to $32-150$ ps. ${ }^{18}$ Therefore, a further decrease of the switching time beyond the value observed here is feasible by the use of current pulses with shorter $T_{\mathrm{p}}$ and $\Delta T$ and by the further optimization of the FEA device structure; for example, the reduction of the gate area and the device capacitance.

In summary, we demonstrated the sub-nanosecond switching of field emission current using a nano-fabricated metallic field-emitter array cathode in a high-acceleration gradient environment and the acceleration of the field emission electron bunch up to $5 \mathrm{MeV}$, using a combined diode$\mathrm{RF}$ cavity electron gun. In contrast to the previously reported $\mathrm{RF}$ electric field excitation of the field emission, ${ }^{7,8,12,24}$ the sub-nanosecond field emission switching method demonstrated here operates in single-pulse mode. This can open up different application areas such as the realization of compact single-pulse microwave/ $\mathrm{THz}^{25}$ or hard $\mathrm{x}$-ray radiation sources $^{26}$ with sub-nanosecond time resolution.

The authors acknowledge B. Haas and J. Leemann for the technical support for the FEA fabrication. This work is partially supported by the Swiss National Science Foundation.

${ }^{1}$ W. P. Dyke and W. W. Dolan, Adv. Electron. Electron Phys. 8, 90 (1956). ${ }^{2}$ C. A. Brau, Nucl. Instr. Method. Phys. Res. A 407, 1 (1998).

${ }^{3}$ C.-M. Tang, A. C. Ting, and T. Swyden, Nucl. Instrum Methods Phys. Res. A 318, 353 (1992).

${ }^{4}$ J. P. Calame, H. F. Gray, and J. L. Shaw, J. Appl. Phys. 73, 1485 (1993).

${ }^{5}$ H. Makishima, S. Miyano, H. Imura, J. Matsuoka, H. Takemura, and A. Okamoto, Appl. Surf. Sci. 147, 230 (1999).

${ }^{6}$ D. R. Whaley, B. M. Gannon, C. R. Smith, C. M. Armstrong, and C. A. Spindt, IEEE Trans. Plasma Sci. 28, 727 (2000).

${ }^{7}$ D. R. Whaley, B. M. Gannon, V. O. Heinen, K. E. Kreischer, C. E. Holland, and C. A. Spindt, IEEE Trans. Plasma Sci. 30, 998 (2002).

${ }^{8}$ K. B. K. Teo, E. Minoux, L. Hudanski, F. Peauger, J.-P. Schnell, L. Gangloff, P. Legagneux, D. Dieumegard, G. A. J. Amaratunga, and W. I. Milne, Nature (London) 437, 968 (2005).

${ }^{9}$ D. A. Kirkpatrick, G. L. Bergeron, M. A. Czarnaski, R. C. Davidson, H. P. Freund, J. J. Hickman, A. Mankofsky, K. T. Tsang, M. Schnur, M. Levinson, and B. M. Ditchek, Nucl. Instrum. Methods Phys. Res. A 318, 349 (1992). 
${ }^{10}$ K. Mima, T. Taguchi, N. Ohigashi, Y. Tsunawaki, M. Shiho, S. Kuruma, M. Fujita, K. Imasaki, C. Yamanaka, and S. Nakai, Nucl. Instrum. Methods Phys. Res. A 341, AB 103 (1994).

${ }^{11}$ H. Ishizuka, S. Kawasaki, M. Arai, H. Shimawaki, K. Yokoo, H. Kubo, A. Watanabe, M. Shiho, and J. Itoh, Nucl. Instrum. Methods Phys. Res. A 393, 479 (1997).

${ }^{12}$ T. Inoue, S. Miyamoto, S. Amano, and T. Mochizuki, Nucl. Instrum. Methods Phys. Res. A 528, 402 (2004).

${ }^{13}$ R. Ganter, R. Bakker, C. Gough, S. C. Leemann, M. Paraliev, M. Pedrozzi, F. Le Pimpec, V. Schlott, L. Rivkin, and A. Wrulich, Phys. Rev. Lett. 100, 064801 (2008).

${ }^{14}$ S. C. Leemann, A. Streun, and A. F. Wrulich, Phys. Rev. ST Accel. Beams. 10, 071302 (2007).

${ }^{15}$ E. Kirk, S. Tsujino, T. Vogel, K. Jefimovs, J. Gobrecht, and A. Wrulich, J. Vac. Sci. Technol. B 27, 1813 (2009).

${ }^{16}$ S. Tsujino, P. Helfenstein, E. Kirk, T. Vogel, C. Escher, and H.-W. Fink, IEEE Electron Device Lett. 31, 1059 (2010).
${ }^{17}$ P. Helfenstein, E. Kirk, K. Jefimovs, T. Vogel, C. Escher, H.-W. Fink, and S. Tsujino, Appl. Phys. Lett. 98, 061502 (2011).

${ }^{18}$ S. Tsujino, M. Paraliev, E. Kirk, T. Vogel. F. Le Pimpec, C. Gough, S. Ivkovic, and H.-H. Braun, J. Vac. Sci. Technol. 29, 02B117 (2011).

${ }^{19}$ M. Paraliev, C. Gough, and S. Ivkovic, in Proceedings of the IEEE International Power Modulator Conference and High Voltage Conference, Las Vegas, NV, May 27-31, 2008.

${ }^{20}$ J.-Y. Raguin, K. Li, R. Bakker, A. Oppelt, and M. Pedrozzi, Nucl. Instrum. Methods. Phys. Res. A 593, 125 (2008).

${ }^{21}$ I. Brodie and C. A. Spindt, Adv. Electron. Electron Phys. 83, 6 (1992).

${ }^{22}$ W. Shockley, J. Appl. Phys. 9, 35 (1938).

${ }^{23}$ S. Ramo, Proc. IRE, p. 583 (September, 1939).

${ }^{24}$ F. M. Charbonnier, J. P. Barbour, L. F. Garrett, and W. P. Dyke, Proc. IEEE, p. 991 (July, 1963).

${ }^{25}$ J. H. Booske, Phys. Plasmas. 15, 05502 (2008).

${ }^{26}$ F. M. Charbonnier, J. P. Barbour, and W. P. Dyke, Radiology. 117, 165 (1975). 